\title{
Planar mesh-lens arrays for millimeter and sub-mm wave focal planes
}

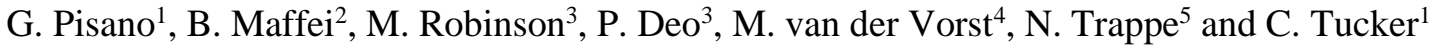 \\ ${ }^{1}$ Cardiff University, Cardiff, CF24 3AA, UK \\ ${ }^{2}$ IAS, Université Paris-Sud, Orsay, 91405, France \\ ${ }^{3}$ The University of Manchester, Manchester, M13 9PL, UK \\ ${ }^{4}$ ESTEC, European Space Agency, Noordwijk, Netherlands \\ ${ }^{5}$ NUI Maynooth, Co. Kildare, Ireland
}

\begin{abstract}
Traditional focal plane arrays for millimeter and sub-millimeter wave instrumentation are designed using corrugated horn antennas. The next generation of experiments dedicated to the detection of Cosmic Microwave Background $(\mathrm{CMB})$ polarization will require large sensitivities and therefore a large number of pixels in their focal planes. In the context of the technology development for future Cosmic Microwave Background satellite missions, we report the preliminary results of the development of a planar array of small lenses based on the mesh-filter technology.
\end{abstract}

\section{INTRODUCTION}

$\mathrm{T}$ HE detection of the CMB polarization B-modes is one of the most challenging current targets in Cosmology. Due to the high sensitivity requirements, the technology development in this field is leading to the realization of compact focal plane arrays with a large number of detectors. The traditional approach based on corrugated horn antennas is not applicable anymore due to the large masses, dimensions and costs involved with this technology.

Alternative solutions have been already employed in different experiments. Some of these are based on the so called 'lenslet arrays'. The manufacture of these small Silicon lenses can be quite complex as they are individually micromachined, anti-reflection coated and then assembled in arrays $[1,2]$

Here we describe a completely different and novel approach based on the mesh-filters technology. These metamaterial structures, made of stacks of copper grids embedded in polypropylene, can be designed to function as filters [3,4], birefringent materials $[5,6,7]$ or as planar focusing surfaces: mesh-lenses [8].

\section{DESIGN}

A mesh-lens working across the W-band $(75-110 \mathrm{GHz})$ with a diameter of $54 \mathrm{~mm}$ and able to accurately focus the radiation within an f/3 beam has already been developed [8]. The working principle of this device is based on the discretization of the lens surface into individual pixelcolumns. Each pixel, consisting of a stack of ten capacitive grids, is designed to provide maximum transmission while inducing local phase-shifts. The incoming phase-front is modified in the same way it would be by a classical lens.

Each pixel column, consisting of a stack of capacitive elements (copper squares) embedded within a polypropylene medium at specific distances, can be modelled as an individual transmission line. The geometry of the single squares is optimized to ideally achieve unitary transmission and a specific differential phase-shit from the lens central pixel. The details of this procedure are described in [8].
We have extended the previous work by designing an array of seven small mesh-lenses. Each lens has a diameter of $\sim 3.2 \lambda$ (for the central frequency) and has a relatively high focusing power of $\sim \mathrm{f} / 0.4$ (Fig.1). Although these arrays will eventually work with planar antennas, here we have designed them to be coupled to a waveguide probe antenna in order to prove the working principle and experimentally measure their performance more easily.

The antenna consists of an open circular waveguide surrounded by a circular choke. Its beam was computed using finite-element analysis software [9] and a cut of its phasefront, at the level of the mesh lens bottom surface, was used as input for the mesh-lens design code. The code produced a set of inhomogeneous grids optimized to modify the incoming curved phase-front into a flat and finite parallel phase-front.

Finite-element analysis was used to simulate the whole system, i.e. waveguide-antenna plus mesh-lens. Fig.2 shows the electromagnetic fields propagating from the antenna through the lens at $90 \mathrm{GHz}$, where the antenna wave-front is modified and flattened by the lens.

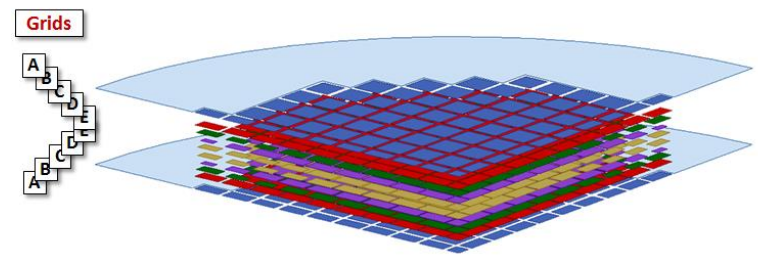

Fig. 1. Mesh-lens CAD model used for the finite-element simulations.

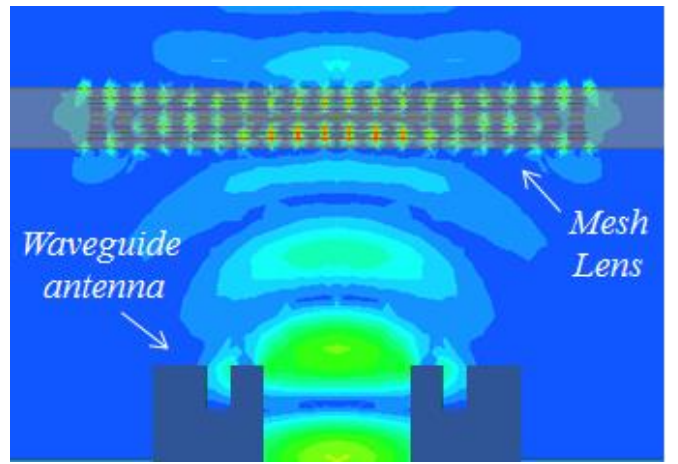

Fig. 2. Waveguide antenna and mesh-lens finite-element simulations at 90 $\mathrm{GHz}$.

\section{MANUFACTURE}

A first mesh-lens array prototype operating across the whole W-band was manufactured. The array, shown in Fig.3, has a thickness of $2.2 \mathrm{~mm}$ and each lens has a diameter of $\sim 10.3 \mathrm{~mm}$ with separation of $\sim 11.3 \mathrm{~mm}$. The array was manufactured using the mesh filters procedures [3]. The only 
additional requirement in their production is the alignment of the grids which needs to guarantee the proper functioning of each pixel-column within each lens.

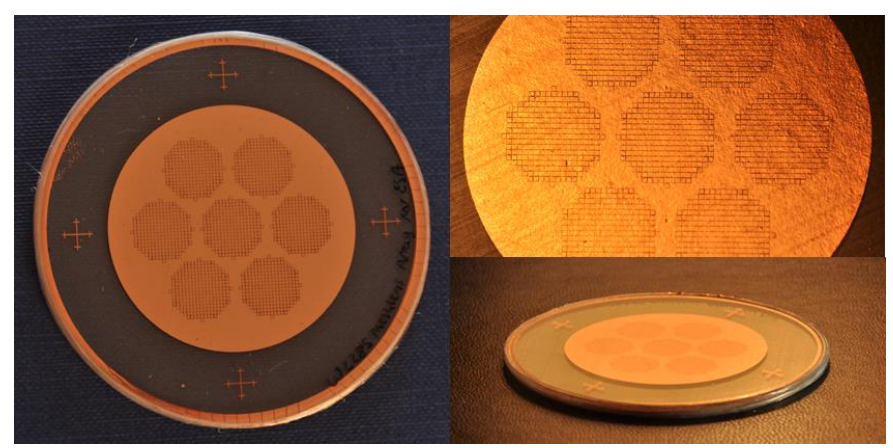

Fig. 3. Seven-pixel mesh-lens array prototype operating across the W-band. Each single lens has a diameter of $\sim 10 \mathrm{~mm}$.

\section{RESULtS}

The experimental tests were performed with a Rohde and Schwarz ZVA40 Vector Network Analyser (VNA) equipped with two W-band converters. The setup was similar to the one described in detail in [8]. In the present case the waveguide probe antenna is mounted on the transmitting head while a corrugated horn is used on the receiving one.

The beam of the probe antenna was measured by rotating the transmitting system around the antenna aperture. An example of $90 \mathrm{GHz}$ beam cut measurements across the $\mathrm{H}$ plane is shown in Fig.4 together with the model expectations. We notice the antenna large beam width, of the order of $\sim 53^{\circ}$ FWHM.

The probe was then accurately aligned and positioned at a distance of $5 \mathrm{~mm}$ from the center of each lens. The transmitting VNA head with the probe-lens system was able to rotate around an axis along the mesh-lens aperture. The H-plane beam cut measurement at $90 \mathrm{GHz}$ for the mesh-lens is reported in Fig.4 together with the finite-element simulations. There is very good agreement between data and predictions showing that the addition of the lens narrows the beam down to $\sim 23^{\circ}$ FWHM. The slight differences at the side-lobe level could be attributed to the accuracy of the alignment and positioning the probe, and to some potential reflections effects.

\section{SUMMARY}

A novel concept of flat lenslet arrays was experimentally proven by using the mesh technology. A 7-elements W-band mesh-lens array, designed to be coupled to a waveguide probe, was manufactured and successfully tested. These encouraging development and results are due to be followed up by an optimization of our lenses to be coupled with planar antennas.

We want to emphasize the fact that these mesh-lens arrays can be designed to be coupled to any types of antenna. To do so, the design phase only requires the field distribution of the antenna at the input surface of the lens. Moreover, the number of processes required during the manufacture is the same for small and large arrays (up to diameters of $\sim 30 \mathrm{~cm}$ ).

This work was supported by the ESA funded project ITT 17393/12/NL/MH, described in [10], and by the Science and Technology Facilities Council (STFC) consolidated grant ST/N000706/1 awarded in Cardiff.

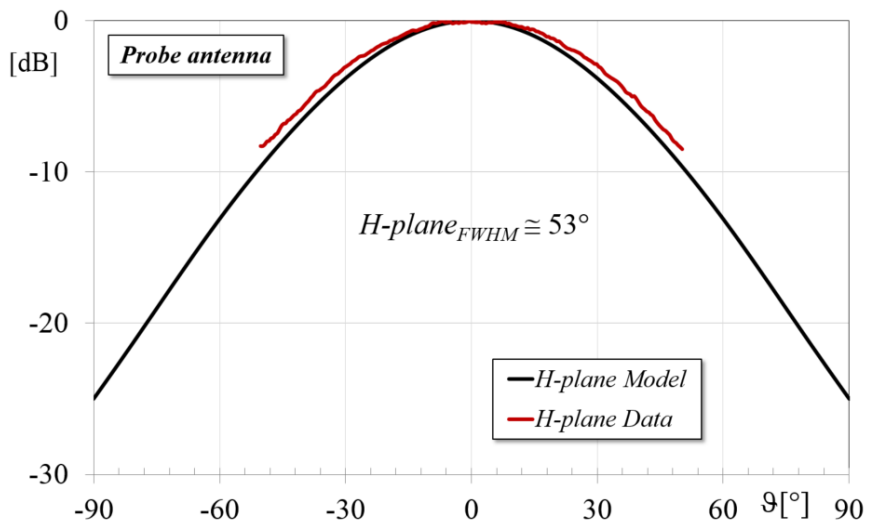

Fig. 4. Waveguide probe antenna H-plane beam model and experimental measurements at $90 \mathrm{GHz}$.

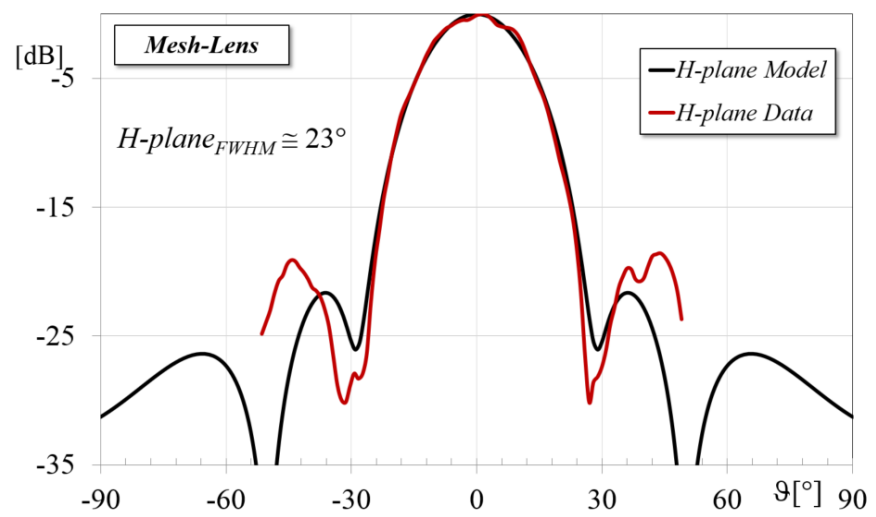

Fig. 5. Mesh-lens H-plane beam model and experimental measurements at 90 GHz.

\section{REFERENCES}

[1] Arnold, K., Ade, P.A., Anthony, A.E., Barron, D., Boettger, D., Borrill, J., Chapman, S., Chinone, Y., Dobbs, M.A., Errard, J. and Fabbian, G., 2012, September. "The bolometric focal plane array of the POLARBEAR CMB experiment," Proc. SPIE vol.8452, pp. 84521D-84521D (2012).

[2] Suzuki, A., Ade, P., Akiba, Y., Aleman, C., Arnold, K., Baccigalupi, C., Barch, B., Barron, D., Bender, A., Boettger, D. and Borrill, J., 2015. "The Polarbear-2 and the Simons Array Experiments." Journal of Low Temperature Physics, pp.1-6.

[3] P. A. R. Ade, G. Pisano, C. E. Tucker and S. O. Weaver, "A review of metal mesh filters," Proc. SPIE, vol. 6275, p. 6275U, 2006.

[4] G. Pisano, P. Ade, C. Tucker, P. Moseley and M.W. Ng, "Metal mesh based metamaterials for millimetre wave and $\mathrm{THz}$ astronomy applications," Proc. 8th UCMMT-2015 Workshop (2016)

[5] G. Pisano, G. Savini, P. Ade and V. Haynes, "A Metal-mesh Achromatic Half-Wave Plate for use at Submillimetre Wavelengths," Applied Optics, vol.47, n.33, pp.6251-6256 (2008)

[6] G. Pisano, M. W. Ng, V. Haynes and B. Maffei, "A broadband metal-mesh half wave plate for millimetre wave linear polarisation rotation," Progress In Electromagnetics Research M, vol. 25, pp. 101-114, 2012.

[7] G. Pisano, B. Maffei, M.W. Ng, V.Haynes, M. Brown, F. Noviello, P. de Bernardis, S.Masi, F.Piacentini, L. Pagano, M. Salatino, B. Ellison, M. Henry, P. de Maagt, B. Shortt, "Development of Large Radii Half-Wave Plates for CMB satellite missions," Proc. SPIE, vol. 9153, id. 91531716 pp. (2014)

[8] G. Pisano, M. W Ng, B. Maffei and F. Ozturk, "A dielectrically embedded flat mesh lens for millimetre waves applications," App. Opt., vol. 52, n.11, pp. 2218-2225, 2013.

[9] High Frequency Structure simulator (HFSS): www.ansys.com

[10] N. Trappe, M. Bucher, P. De Bernardis, J. Delabrouille, P. Deo, M. DePetris, S. Doherty, A. Ghribi, M. Gradziel, L. Kuzmin, B. Maffei, S. Mahashabde, S. Masi, J. A. Murphy, F. Noviello, C. O'Sullivan, L. Pagano, F. Piacentini, M. Piat, G. Pisano, M. Robinson, R. Stompor, A. Tartari, M. van der Vorst, P. Verhoeve, "Next generation sub-millimeter wave focal plane array coupling concepts: an ESA TRP project to develop multichroic focal plane pixels for future CMB polarization experiments,"Proc. SPIE Astronomical Telescopes + Instrumentation (2016) 